\title{
Direct imaging of current induced magnetic vortex gyration in an asymmetric potential well
}

\author{
Andre Bisig, ${ }^{1,2}$ Jan Rhensius, ${ }^{1,3}$ Matthias Kammerer, ${ }^{2}$ Michael Curcic, ${ }^{2}$ Hermann Stoll, ${ }^{2}$ \\ Gisela Schütz, ${ }^{2}$ Bartel Van Waeyenberge, ${ }^{4}$ Kang Wei Chou, ${ }^{5}$ Tolek Tyliszczak, ${ }^{5}$ \\ Laura J. Heyderman, ${ }^{3}$ Stephen Krzyk, ${ }^{1}$ Arndt von Bieren, ${ }^{1}$ and Mathias Kläui ${ }^{1, a)}$ \\ ${ }^{1}$ Fachbereich Physik, Universität Konstanz, Universitätsstraße 10, 78457 Konstanz, Germany \\ ${ }^{2}$ Max-Planck-Institut für Metallforschung, Heisenbergstrasse 3, 70569 Stuttgart, Germany \\ ${ }^{3}$ Laboratory for Micro- and Nanotechnology, Paul Scherrer Institut, 5232 Villigen PSI, Switzerland \\ ${ }^{4}$ Department of Solid State Sciences, Ghent University, Proeftuinstraat 86, 9000 Ghent, Belgium \\ ${ }^{5}$ Advanced Light Source, LBNL, Berkeley, California 94720, USA
}

(Received 20 November 2009; accepted 5 March 2010; published online 16 April 2010)

\begin{abstract}
Employing time-resolved $\mathrm{x}$-ray microscopy, we investigate the dynamics of a pinned magnetic vortex domain wall in a magnetic nanowire. The gyrotropic motion of the vortex core is imaged in response to an exciting ac current. The elliptical vortex core trajectory at resonance reveals asymmetries in the local potential well that are correlated with the pinning geometry. Using the analytical model of a two-dimensional harmonic oscillator, we determine the resonance frequency of the vortex core gyration and, from the eccentricity of the vortex core trajectory at resonance, we can deduce the stiffness of the local potential well. (C) 2010 American Institute of Physics. [doi:10.1063/1.3373590]
\end{abstract}

Electrons traveling through ferromagnets with inhomogeneous magnetization act via the spin-transfer-torque on the local magnetization, which leads to current induced domain wall motion ${ }^{1,2}$ or domain wall excitation, such as oscillations of domain walls ${ }^{3-6}$ or the excitation of magnetic vortices. ${ }^{7,8}$ Such a control of magnetic domain walls or vortices by electrical currents opens the possibility to store information in nonvolatile devices. ${ }^{9}$

Domain walls in magnetic nanowires often exhibit an energetically favorable vortex structure in which the magnetization curls in the plane of the wire around a central point, called the vortex core, where the magnetization points out-of-plane. ${ }^{10}$ This small region, only $\approx 10-20 \mathrm{~nm}$ in diameter, ${ }^{11,12}$ controls the dynamics of the whole vortex domain wall. The out-of-plane component of the core magnetization, the polarity $p$, can either point up, $p=+1$, or down, $p=-1$, and the chirality $c$ is defined by the sense of the curling of the in plane magnetization, either counterclockwise $(c=+1)$ or clockwise $(c=-1)$. Magnetic fields as well as spin-polarized currents interacting with the domain wall can induce a gyration of the vortex core. While the sense of rotation of the vortex core gyration is solely determined by the core polarity $p$, the phase with respect to the excitation depends on the excitation type (adiabatic spin-torque, nonadiabatic spin-torque, or magnetic field) and also on the chirality $c .^{13-15}$

So far, current induced vortex core gyration has been studied by electrical detection of the resonant response of the magnetic vortex core of a vortex domain wall trapped at a local confinement in a magnetic nanowire. ${ }^{4-6}$ While the field and current induced gyration in symmetric elements with symmetric potential wells has been studied, ${ }^{8}$ the more relevant case of asymmetric potential wells, occurring for pinned domain walls, has not been revealed. In particular, a

\footnotetext{
${ }^{a)}$ Electronic mail: mathias@klaeui.de. Also at: Zukunftskolleg, Universität Konstanz, 78457 Konstanz, Germany.
}

direct observation of the vortex core trajectory in local asymmetric potential wells is missing and this is all the more important since the vortex core velocity is no longer constant, but varies with respect to its position, depending on the local potential geometry. Additionally, both field and current driven vortex core motion has been observed in disks and squares, but investigations of pure current induced gyration is difficult, because of the Oersted fields generated by the inhomogeneous current flow through the contacts. ${ }^{8}$ In the case of disks or squares, the contacts are overlapping with the elements, leading to higher Oersted fields, while in the nanowire geometry, where the contacts are placed far away from the area under investigation, Oersted field contributions are minimized.

Here we report on the experimental observation of vortex core gyration of a pinned vortex domain wall in a confined magnetic nanowire. Employing time-resolved scanning transmission X-ray microscopy (STXM), we image the vortex core gyration trajectory, exciting with an ac current injected into the wire. The elliptical local potential is revealed by the eccentricity of the core trajectory. Following the twodimensional harmonic oscillator model, based on the extended Thiele equation including spin-torque terms, we determine the local potential shape. Finally finite element calculations are carried out to calculate the local current density at the vortex core and show that there are only minor contributions to the excitation due to the Oersted field.

We study magnetic vortex domain walls in a $50 \mathrm{~nm}$ thick and $500 \mathrm{~nm}$ wide circular permalloy $\left(\mathrm{Ni}_{80} \mathrm{Fe}_{20}\right)$ nanowire (radius $5 \mu \mathrm{m}$ ), where the domain wall is pinned by an artificial notch at the center of the wire, see Fig. 1(a). The wire is fabricated on top of a $100 \mathrm{~nm} \mathrm{Si}_{3} \mathrm{Ni}_{4}$ membrane by electron beam lithography, molecular beam evaporation in UHV and lift-off processing. To improve cooling, a $150 \mathrm{~nm}$ thick aluminum nitride layer was deposited on top of the structures and a $120 \mathrm{~nm}$ thick aluminum layer was sputtered on the back of the sample. The wires are contacted by $\mathrm{Cr}(7 \mathrm{~nm}) /$ 


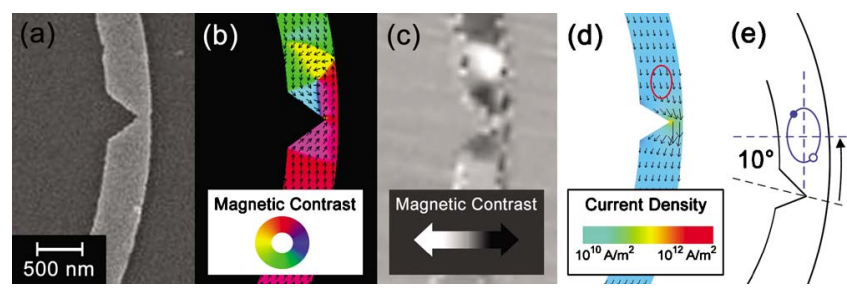

FIG. 1. (Color online) (a) Scanning electron microscope image of the permalloy nanowire. (b) Micromagnetic simulation of the relaxed magnetic vortex domain wall pinned at the wire constriction. (c) STXM image of the nanowire under investigation showing XMCD contrast. (d) Calculated current distribution in the wire, showing a homogeneous current flow in the region of vortex gyration. (e) Schematic illustration of the experimental geometry; the wire itself is tilted by $-10^{\circ}$ with respect to the horizontal axis.

$\mathrm{Au}(25 \mathrm{~nm})$ contacts at a distance larger than $4 \mu \mathrm{m}$ from the notch. Figures 1(b) and 1(c) compare magnetic images of a pinned vortex domain wall employing STXM with micromagnetic simulations showing the relaxed state of a pinned vortex domain wall in the same geometry. Inhomogeneous currents flowing vertically from the contacts into the wire generate an in-plane Oersted field transverse to the wire. Finite element calculations carried out using COMSOL multiphysics ${ }^{16}$ show, that the resulting in-plane field component at the position of the artificial notch is negligible $\left(\begin{array}{lll}H<2 & \mu \mathrm{T}\end{array}\right)$ at a constant current density of $j_{e}=9.6$ $\times 10^{10} \mathrm{~A} / \mathrm{m}^{2}$ in the permalloy wire near the electrical contacts. Furthermore, these calculations show that the current is flowing homogeneously in the area of the vortex core gyration, see Fig. 1(d). Thus, this geometry is well-suited to study current induced gyration of the vortex core by the application of high density sinusoidal currents.

The time dependent vortex core trajectory is imaged by a stroboscopic measurement technique employing STXM at the Advanced Light Source (ALS, Beamline 11.0.2). ${ }^{17}$ The lateral resolution is $\approx 30 \mathrm{~nm}$. The sample is aligned perpendicular to the $\mathrm{x}$-ray beam. Therefore we are only sensitive to the out-of-plane component of the magnetization, which is for our samples the vortex core. Alternatively the sample can be tilted by $30^{\circ}$, which allows the imaging of the in-plane magnetization contrast. This data is recorded at the Ni $\mathrm{L}_{3}$-absorption edge $(852.7 \mathrm{eV})$.

The magnetic response to an external excitation is imaged by the individual photon flashes of the synchrotron, with a time resolution given by the width of the x-ray photon flashes $(70 \mathrm{ps})$. The vortex core is imaged at 25 different phases in response to the injected sinusoidal current $j(t)$ $=9.6 \times 10^{10} \mathrm{~A} / \mathrm{m}^{2} \sin (\omega t+\phi)$. The external excitation frequency $f=\omega / 2 \pi$ is locked to the synchrotron orbit clock and can be varied in steps of $20 \mathrm{MHz}$. These frequencies are given by the $500 \mathrm{MHz}$ probing rate of the synchrotron divided by the 25 channel frame acquisition. By using the capacitive coupling of the exciting current to the APD, the phase $\phi$ of the excitation is determined relative to the phase $\Phi$ of the magnetic response: $\Phi=\phi+\phi_{0}$, where $\phi_{0}$ is unknown but constant. The temporal error of the relative phase is proportional to the excitation frequency $f$ and is of the order of 50-90 ps.

For various frequencies $f$ between 240 and $460 \mathrm{MHz}$, the gyrotropic resonance of the vortex core is imaged and the relative phase of the gyration $\Phi$ is measured. Figure 2 compares the measured relative phase of the gyration $\Phi$ with the

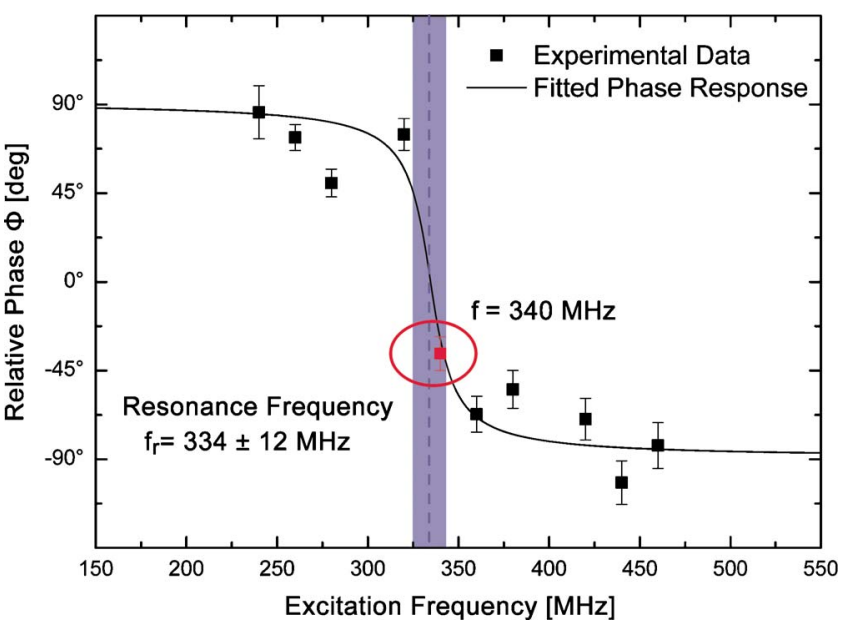

FIG. 2. (Color online) Experimental data of the relative phase $\Phi$ of the vortex core gyration due to ac current injection $j(t)=9.6$ $\times 10^{10} \mathrm{~A} / \mathrm{m}^{2} \sin (2 \pi f t+\phi)$ with a constant phase $\phi$. The fitted phase response corresponds to a resonance frequency $f_{r}=334 \pm 12 \mathrm{MHz}$, indicated by the blue vertical bar. The corresponding gyration trajectory for an excitation frequency of $340 \mathrm{MHz}$, close to the resonance frequency (see red datapoint), is shown in Fig. 3.

fitted phase response. The resonance frequency $f_{r}$ is determined to be $334 \pm 12 \mathrm{MHz}$. In Fig. 3(a), the vortex core trajectory at $340 \mathrm{MHz}$ near the resonance frequency $f_{r}$ is plotted. We measure an eccentricity of $e=\sqrt{A_{y}^{2}-A_{x}^{2}} / A_{y}$ $=0.77 \pm 0.04$ for the elliptical trajectory and a tilt angle $\theta$ of $10^{\circ}$ with respect to the notch $\left(\theta=10.1^{\circ} \pm 0.5^{\circ}\right)$, see Fig. 1(e). The clockwise sense of gyration is dictated by the negative vortex core polarity, here $p=-1$. The elliptical trajectory at resonance indicates a strong asymmetry of the pinning potential, due to the pinning of the vortex domain wall by the artificial notch.

To analyze these results, numerical calculations are performed based on the Thiele equation including the spintorque-terms as follows: $:^{13,18}$

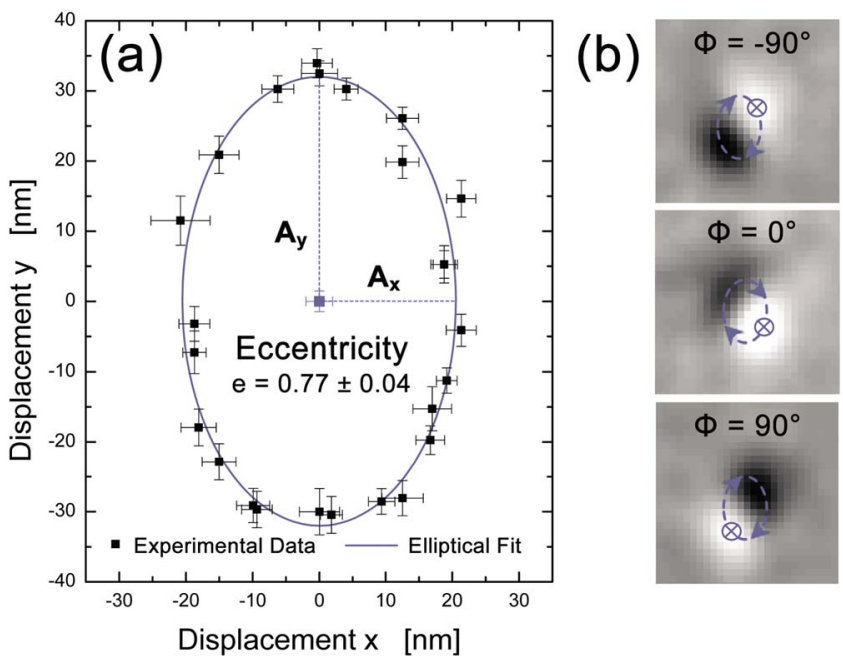

FIG. 3. (Color online) (a) The experimentally determined vortex core trajectory at $f=340 \mathrm{MHz}$ near the resonance frequency $f_{r}$. The ellipse is fitted through the data points and its eccentricity is a measure of the local pinning potential well. (b) Differential STXM snapshots indicate elliptical gyration of the vortex core under continuous ac excitation with $340 \mathrm{MHz}$. To remove nonmagnetic contrast, two images with out-of-plane XMCD contrast recorded at $180^{\circ}$ phase difference are divided by each other. 


$$
\vec{\nabla}_{\mathbf{r}} V(\mathbf{r})+\mathbf{G} \times[\mathbf{u}(t)-\dot{\mathbf{r}}]+D \cdot[\alpha \dot{\mathbf{r}}-\beta \mathbf{u}(t)]=0,
$$

where $\mathbf{r}=(x, y)$ describes the position of the vortex core. Following our experimental observation of an elliptical trajectory, we assume a paraboloidal potential well $V(\vec{r})=\kappa_{x} x^{2} / 2$ $+\kappa_{y} y^{2} / 2$, where $\kappa_{x}$ and $\kappa_{y}$ are the effective stiffness coefficients. ${ }^{4,19}$ This approximation is valid for small vortex core displacements around its equilibrium position. The gyrovector $\mathbf{G}=p G \hat{\mathbf{e}}_{\mathbf{z}}$, with $G=2 \pi M_{S} \mu_{0} t / \gamma$ is pointing out-ofplane, $t$ and $M_{S}$ are the thickness and the saturation magnetization of the wire, respectively, and $\gamma$ is the gyromagnetic ratio. The external force is described by the spin drift velocity $\mathbf{u}(t)=\left(j_{e} P \mu_{B} / e M_{S}\right) \hat{\mathbf{e}}_{\theta} \cdot \sin (2 \pi f t+\phi)$, where $j_{e}$ is the current density and $\mathrm{P}$ its spin polarization, $e$ is the positive elementary charge, and $\mu_{B}$ the Bohr magneton. The damping tensor $D$ is diagonal for the case of a disk ${ }^{20}$ and its components are given by $D_{x y}=G\left[1+1 / 2 \ln \left(R_{V} / \delta_{V}\right)\right] \delta_{x y}$, where $2 R_{V}=590 \mathrm{~nm}$ is the domain wall width and $\delta_{V}=10 \mathrm{~nm}$ is the vortex core radius. ${ }^{11}$ The parameter $\alpha=0.005$ is the Gilbert damping of permalloy and $\beta=2 \alpha$ is assumed for the nonadiabaticity of the spin-torque. ${ }^{3,21}$ Note that the harmonic approximation holds only for small oscillations at low excitation power because recent experiments indicate nonharmonic contributions to the local potential at higher power. $^{6}$

This two-dimensional harmonic oscillator model has been solved analytically for sinusoidal excitation and any harmonic potential by Buchanan et al. ${ }^{19}$ and has been extended by Moriya et al. ${ }^{4}$ for current induced excitations. The steady state solution has the form of an elliptical trajectory as follows:

$$
\begin{aligned}
& \left(x-x_{0}\right)=A_{x} \sin \left(2 \pi f t+\epsilon_{x}\right), \\
& \left(y-y_{0}\right)=A_{y} \sin \left(2 \pi f t+\epsilon_{y}\right),
\end{aligned}
$$

where $A_{x}\left(\kappa_{x}, \kappa_{y}, f\right)$ in our case is the minor axis and $A_{y}\left(\kappa_{x}, \kappa_{y}, f\right)$ is the major axis. Both depend on the excitation frequency and reflect the strength of the pinning potential $V(\mathbf{r})$. The two phases $\epsilon_{x}\left(\kappa_{x}, \kappa_{y}, p, f\right)$ and $\epsilon_{y}\left(\kappa_{x}, \kappa_{y}, p, f\right)$ furthermore depend on the vortex core polarity $p$. The resonance frequency in this model is

$$
f_{r}=\frac{\omega_{r}}{2 \pi}=\frac{G \sqrt{\kappa_{x} \kappa_{y}}}{G^{2}+\alpha^{2} D^{2}},
$$

giving a relation between $\kappa_{x}$ and $\kappa_{y}$ for $f_{r}=334 \mathrm{MHz}$. The ratio

$$
r\left(\kappa_{x}, \kappa_{y}, f_{r}\right)=\frac{A_{x}\left(\kappa_{x}, \kappa_{y}, f_{r}\right)}{A_{y}\left(\kappa_{x}, \kappa_{y}, f_{r}\right)},
$$

gives another relationship between $\kappa_{x}$ and $\kappa_{y}$ at the resonance frequency. From Eqs. (4) and (5) it is then possible to unambiguously determine $\kappa_{x}$ and $\kappa_{y}$. The measured vortex core trajectory at $340 \mathrm{MHz}$, close to the resonance frequency $f_{r}=334 \mathrm{MHz}$, has amplitudes $A_{x}\left(\kappa_{x}, \kappa_{y}, f_{r}\right)=20.5 \pm 1.2 \mathrm{~nm}$ and $A_{y}\left(\kappa_{x}, \kappa_{y}, f_{r}\right)=32.0 \pm 0.9 \mathrm{~nm}$. With these values the potential stiffness coefficients can be determined as $\kappa_{x}$ $=4.7 \pm 0.4 \times 10^{-3} \mathrm{~kg} / \mathrm{s}^{2}$ and $\kappa_{y}=1.9 \pm 0.2 \times 10^{-3} \mathrm{~kg} / \mathrm{s}^{2}$.

Note that the absolute measured values of the amplitudes $A_{x}$ and $A_{y}$ are smaller than the amplitudes calculated using this model; $A_{x}=66 \mathrm{~nm}$ and $A_{y}=103 \mathrm{~nm}$, respectively. This can be attributed to the fact that the absolute amplitudes at the resonance peak are very sensitive to the excitation frequency. Furthermore, due to reflections of the microwave injection, the effective current density $j_{e}$ can be lower than the maximum injected density of $9.6 \times 10^{10} \mathrm{~A} / \mathrm{m}^{2}$.

In conclusion, we have investigated the asymmetric pinning potential for a vortex domain wall employing timeresolved STXM imaging of current-induced vortex core gyration with only minor contributions from the Oersted field. From the gyration amplitudes at the resonance frequency we determine the stiffness $\kappa_{x}$ and $\kappa_{y}$ of the local potential, which opens a way to obtain the full potential landscape.

We thank Lutz Heyne for numerical calculations, the DFG (SFB 767, Grant No. KL1811), the Landesstiftung Baden Württemberg, the European Research Council via its Starting Independent Researcher Grant (Grant No. ERC2007-Stg 208162) scheme, EU RTN SPINSWITCH (Grant No. MRTN-CT-2006-035327), and the Samsung Advanced Institute of Technology for financial support. The Advanced Light Source is supported by the Director, Office of Science, Office of Basic Energy Sciences, of the U.S. Department of Energy under Contract No. DE-AC02-05CH11231.

${ }^{1}$ A. Yamaguchi, T. Ono, S. Nasu, K. Miyake, K. Mibu, and T. Shinjo, Phys Rev. Lett. 92, 077205 (2004).

${ }^{2}$ M. Kläui, C. A. F. Vaz, J. A. C. Bland, W. Wernsdorfer, G. Faini, E. Cambril, L. J. Heyderman, F. Nolting, and U. Rüdiger, Phys. Rev. Lett. 94, 106601 (2005).

${ }^{3}$ L. Thomas, M. Hayashi, X. Jiang, R. Moriya, C. Rettner, and S. S. P. Parkin, Nature (London) 443, 197 (2006).

${ }^{4}$ R. Moriya, L. Thomas, M. Hayashi, Y. B. Bazaly, C. Rettner, and S. S. P. Parkin, Nat. Phys. 4, 368 (2008).

${ }^{5}$ D. Bedau, M. Kläui, S. Krzyk, U. Rüdiger, G. Faini, and L. Vila, Phys. Rev. Lett. 99, 146601 (2007).

${ }^{6}$ D. Bedau, M. Kläui, M. T. Hua, S. Krzyk, U. Rüdiger, G. Faini, and L. Vila, Phys. Rev. Lett. 101, 256602 (2008).

${ }^{7}$ S. Kasai, Y. Nakatani, K. Kobayashi, H. Kohno, and T. Ono, Phys. Rev. Lett. 97, 107204 (2006).

${ }^{8}$ M. Bolte, G. Meier, B. Krüger, A. Drews, R. Eiselt, L. Bocklage, S. Bohlens, T. Tyliszczak, A. Vansteenkiste, B. Van Waeyenberge, and K. W. Chou, Phys. Rev. Lett. 100, 176601 (2008).

${ }^{9}$ S. S. P. Parkin, M. Hayashi, and L. Thomas, Science 320, 190 (2008).

${ }^{10}$ M. Kläui, J. Phys.: Condens. Matter 20, 313001 (2008).

${ }^{11}$ A. Wachowiak, J. Wiebe, M. Bode, O. Pietzsch, M. Morgenstern, and R. Wiesendanger, Science 298, 577 (2002).

${ }^{12}$ K. W. Chou, A. Puzic, H. Stoll, D. Dolgos, G. Schütz, B. van Waeyenberge, A. Vansteenkiste, T. Tyliszczak, G. Woltersdorf, and C. H. Back, Appl. Phys. Lett. 90, 202505 (2007).

${ }^{13}$ A. A. Thiele, Phys. Rev. Lett. 30, 230 (1973)

${ }^{14}$ D. L. Huber, J. Appl. Phys. 53, 1899 (1982).

${ }^{15}$ B. Krüger, A. Drews, M. Bolte, U. Merkt, D. Pfannkuche, and G. Meier, Phys. Rev. B 76, 224426 (2007).

${ }^{16}$ COMSOL Multiphysics, http://www.comsol.de.

${ }^{17}$ A. L. D. Kilcoyne, T. Tyliszczak, W. F. Steele, S. Fakra, P. Hitchcock, K. Franck, E. Anderson, B. Harteneck, E. G. Rightor, G. E. Mitchell, A. P. Hitchcock, L. Yang, T. Warwick, and H. Ade, J. Synchrotron Radiat. 10, 125 (2003).

${ }^{18}$ A. Thiaville, Y. Nakatani, J. Miltat, and Y. Suzuki, Europhys. Lett. 69, 990 (2005).

${ }^{19}$ K. S. Buchanan, P. E. Roy, F. Y. Fradin, K. Yu. Guslienko, M. Grimsditch, S. D. Bader, and V. Novosad, J. Appl. Phys. 99, 08 C707 (2006); K. S. Buchanan, P. E. Roy, M. Grimsditch, F. Y. Fradin, K. Yu. Guslienko, S. D. Bader, and V. Novosad, Phys. Rev. B 74, 064404 (2006).

${ }^{20}$ K. Yu. Guslienko, Appl. Phys. Lett. 89, 022510 (2006).

${ }^{21}$ S. Zhang and Z. Li, Phys. Rev. Lett. 93, 127204 (2004). 